ranoner

\title{
Evaluación de la persistencia de síntomas luego de un episodio de infección por SARS-CoV-2
}

\author{
Recibido: 14/7/21 Aceptado: 5/11/21
}

Dra. Junnai Carrillo ${ }^{1} \mathbb{B}$, Vanesa Fridman ${ }^{2} \mathbb{C}$, Daniel Stecher $^{3}$ (D).

\section{RESUMEN}

Introducción: Es frecuente que, luego de un episodio de COVID-19, los pacientes persistan con síntomas, lo cual impacta negativamente en su calidad de vida.

El objetivo primario de este estudio es determinar los síntomas prevalentes y su duración luego de un episodio de COVID-19 y la persistencia de estos al mes y a los tres meses del episodio. Los objetivos secundarios son describir el lapso entre el episodio de COVID-19, la reinserción laboral o académica y la reanudación de actividad física.

Material y métodos: Estudio descriptivo, observacional, en adultos con antecedente de infección por SARS-CoV-2, realizado a través de una encuesta en línea. Se relacionaron variables demográficas y comorbilidades con síntomas durante la infección, al mes y al tercer mes del episodio.

Resultados: Participaron 308 personas. Los síntomas más comunes en la fase aguda fueron dolor muscular y fiebre. Al mes, el 89\% presentaba síntomas persistentes, siendo los más frecuentes: fatiga y mialgias. Luego de tres meses, 34,85\% presentaba síntomas, siendo los más frecuentes: fatiga y anosmia. Solo el $45,7 \%$ de las personas logró la reinserción laboral/académica y el 17\% de los individuos logró reiniciar actividad física al alta epidemiológica.

Discusión: El porcentaje de personas con síntomas persistentes después de un episodio de COVID-19 es alto. La persistencia de los síntomas disminuye con el tiempo, lo cual es un dato alentador para todos aquellos pacientes que consultan por continuar sintomáticos.
1 Médica Residente de tercer año, Enfermedades Infecciosas, División Infectología, Departamento de Medicina Interna, Hospital de Clínicas "José de San Martín", Universidad de Buenos Aires, Argentina. Av. Córdoba 2351. Piso 3, Sala 2. CP 1120 CABA. junncarrillo@outlook.com

${ }^{2}$ Médica Especialista en Enfermedades Infecciosas, Médica de Planta, División Infectología, Departamento de Medicina Interna, Hospital de Clínicas “José de San Martín", Universidad de Buenos Aires, Argentina. Av. Córdoba 2351. Piso 3, Sala 2. CP 1120 CABA. vfridman@intramed.net

${ }^{3}$ Médico Especialista en Enfermedades Infecciosas, Jefe de División Infectología, Departamento de Medicina Interna, Hospital de Clínicas "José de San Martín", Universidad de Buenos Aires, Argentina. Av. Córdoba 2351. Piso 3, Sala 2. CP 1120 CABA. dstecher@intramed.net

Institución: División Infectología, Departamento de Medicina Interna, Hospital de Clínicas "José de San Martín", Universidad de Buenos Aires, Argentina.

Ninguno de los autores declara presentar conflicto de intereses en relación a esta publicación.

Palabras clave: post COVID-19, SARS-CoV-2, síntomas persistentes, COVID-19. 


\section{Introducción}

Desde diciembre de 2019, el mundo se encuentra afectado por la propagación de la infección de un nuevo coronavirus, el SARS-CoV-2. Este virus causa principalmente compromiso respiratorio. Las formas de presentación oscilan entre los síntomas leves y la neumonía, que puede llegar a ser severa y requerir de internación en áreas críticas con asistencia respiratoria mecánica. Al momento, no se ha identificado una terapéutica eficaz para disminuir la mortalidad. En Argentina, hasta el 28 de junio de 2021 se han registrado 4.423 .636 casos de COVID-19 desde su aparición en el país y 93.142 personas han fallecido por causa relacionada a dicha enfermedad. No es infrecuente que, luego del episodio agudo y de recibir el alta epidemiológica, los pacientes persistan con síntomas, lo cual impacta negativamente en la calidad de vida de estas personas. Es una consulta frecuente en la práctica clínica actual la prolongada duración de los síntomas, siendo difícil para los profesionales de la salud emitir una respuesta concreta por la falta de datos sobre este tópico.

El objetivo primario del presente trabajo es determinar los síntomas prevalentes y su duración luego de un episodio de infección por SARS-CoV-2 y la persistencia de los mismos al mes y a los tres meses luego del episodio.

Como objetivos secundarios se pretende describir el lapso entre el episodio de COVID-19 y la reinserción laboral o académica, la reanudación de la actividad física, determinar cuáles fueron los síntomas más frecuentes durante la etapa aguda de la infección y describir la relación entre la persistencia de síntomas y diferentes variables como edad, género, necesidad de internación y tratamiento recibido.

\section{Material y métodos}

Se trata de un estudio descriptivo, observacional, que se realizó desde el 2 de noviembre de 2020 hasta el 2 de enero de 2021. Se incluyeron personas mayores de 18 años con diagnóstico de infección por SARSCoV-2 confirmado por PCR a través de un hisopado nasofaríngeo. Se incluyeron individuos en los que había transcurrido por lo menos un mes del diagnóstico. A dichas personas se las contactó a través de correo electrónico o por teléfono (a través de la aplicación Whatsapp $\left.{ }^{\circledR}\right)$ y se les envió un mensaje explicándoles en qué consistía el estudio e invitándolas a participar del mismo. A aquellos individuos que aceptaron, se les envió un formulario para completar en forma remota (se completó en línea a través de la plataforma Google Forms $\left.{ }^{\circledR}\right)$. El cuestionario era anónimo, no incluía ningún dato filiatorio y constaba de diez preguntas de opción múltiple (en algunas de las cuales era válido completar más de una opción).

El formulario incluyó variables demográficas (edad, sexo al nacer); comorbilidades (diabetes mellitus, hipertensión arterial, enfermedad pulmonar crónica, cardiopatías, inmunosupresión congénita o adquirida, insuficiencia renal crónica con o sin necesidad de diálisis, sobrepeso, tabaquismo); datos clínicos al diagnóstico (temperatura axilar mayor o igual a $37.5^{\circ} \mathrm{C}$, tos, falta de aire, fatiga, anosmia, ageusia, odinofagia, mialgias, diarrea); manejo del episodio de infección por SARS-CoV-2, incluyendo internación en clínica médica o unidad de cuidados críticos (UTI), requerimiento de oxigeno por cánula, máscara o asistencia respiratoria mecánica (ARM), y tratamientos recibidos (corticoides, plasma de convaleciente, lopinavir/ ritonavir, hidroxicloroquina, azitromicina). Estas variables no fueron medidas ni confirmadas por los investigadores, sino que fueron referidas como antecedentes por los individuos encuestados.

Se consultó la persistencia de síntomas al mes y a los tres meses del diagnóstico de SARS-CoV-2, incluyendo: fiebre, tos, falta de aire/disnea, fatiga, ageusia, anosmia, odinofagia, diarrea, dolor torácico, déficit de atención, déficit de memoria, trastornos del sueño, disfonía. Al tratarse de un estudio de corte transversal no contempla un periodo de seguimiento sino que se trata de una observación en un punto temporal fijo. Se inquirió sobre la reincorporación a la actividad habitual (laboral/ académica) y reinicio de la actividad física, así como el tiempo desde el diagnóstico hasta el reinicio de la misma.

Se definió el día del inicio de la infección por SARS-CoV-2 al día de aparición de los síntomas y, en los pacientes asintomáticos, al día de la detección del SARS-CoV-2 mediante PCR en hisopado nasofaríngeo. Se definió como alta epidemiológica al día 10 desde el inicio de la infección.

\section{Resultados}

Un total de 308 personas $(P)$ con antecedente de infección por SARS-CoV-2 respondieron las preguntas del formulario digital. Predominó el género femenino (182 
mujeres, 59,5\%), con edad mayoritariamente entre 18 y 35 años (136 P, 44,1\%). De toda la población estudiada, 123 personas $(39,9 \%)$ reportaron enfermedades o condiciones preexistentes, siendo las más frecuentes: sobrepeso en 71 casos $(57,7 \%)$, hipertensión arterial (HTA) en 29 (23,6\%) y asma en 17 (13,8\%) (Tabla 1$)$.

De las 304 personas que respondieron sobre los síntomas durante la fase aguda de infección, se encontró que los más frecuentes fueron dolor muscular/mialgias en 172 $(55,84 \%)$, seguido de fiebre en $158(51,29 \%)$ y cefalea en 154 (50\%) (Tabla 2).

\section{Persistencia de síntomas al mes}

Doscientos setenta y cuatro (89\%) personas refirieron síntomas persistentes al mes del inicio de la infección. Los más frecuentes fueron: fatiga en 151 casos (52,95\%), mialgias en 69 (24,21\%), disnea en 65 (22,80\%), anosmia en 60 (21,05\%), tos en $55(19,29 \%)$, cefalea en $46(16,14 \%)$, disgeusia en 37 (12,98\%), odinofagia en 26 (9,12\%), dolor torácico en $24(8,42 \%)$ y en 11 casos $(3,57 \%)$ se reportó persistencia de fiebre. Solo 11 encuestados $(3,85 \%)$ negaron síntomas persistentes al mes del inicio de la infección. Veintitrés personas reportaron no tener más de un mes desde el inicio de la enfermedad.

Tabla 1. Características de la población

Sexo al nacer

Mujer: 182 (59,5\%)

Hombre: 125 (40,5\%)

Edad

18-35 años: $136(44,1 \%)$

36-50 años: $119(38,6 \%)$

51-65 años: $42(13,6 \%)$

66-75 años: 5 (1,6\%)

Mayor a 75 años: 5 (1,6\%)

Enfermedades preexistentes ( $n=123)$

Sobrepeso: $71(57,7 \%)$

Hipertensión arterial: 29 (23,6\%)

Asma 17: $(13,8 \%)$

Diabetes mellitus: $13(10,6 \%)$

Tabaquismo: 13 (10,6\%)

EPOC: $5(4,1 \%)$

Tratamiento inmunosupresor: $5(4,1 \%)$

Enfermedad oncológica: 2 (1,62\%)

ERC* con requerimiento de hemodiálisis: $1(0,8 \%)$

Infección por VIH: $1(0,8 \%)$

\section{Requerimiento de internación}

En clínica médica: $89(28,9 \%)$

En terapia intensiva: $11(3,5 \%)$

Sin requerimiento de internación: 208 (67,5\%)

Tratamiento durante la internación $(n=100)$

Oxígeno en cánula/máscara: 30 (30\%)

Asistencia respiratoria mecánica: 7 (7\%)

Plasma de convaleciente: 13 (13\%)

Corticoides: $45(45 \%)$

Otros tratamientos (Lopinavir/ritonavir, Azitromicina u otros antibióticos): 48 (48\%)

* ERC: Enfermedad Renal Crónica 
La fatiga al mes del inicio de la enfermedad fue reportada en todos los pacientes con antecedente de EPOC y en más de la mitad de aquellos con DBT, HTA, tratamiento inmunosupresor, sobrepeso y tabaquismo. El $77,2 \%$ de los encuestados que reportaron comorbilidades informaron tener fatiga al mes de la enfermedad.

La disnea estuvo presente al mes de la enfermedad en más del $30 \%$ de los pacientes con antecedente de HTA, diabetes mellitus, sobrepeso y tabaquismo y en el $60 \%$ de los pacientes con antecedente de EPOC.

El síntoma persistente más frecuente al primer mes de la infección en todos los individuos con comorbilidades fue la fatiga, con una prevalencia del $47 \%$ en los pacientes con antecedente de asma, $54 \%$ en individuos con diabetes mellitus, $65,5 \%$ en aquellos con hipertensión arterial, $100 \%$ en quienes presentaban antecedente de EPOC, $80 \%$ en los que reciben tratamiento inmunosupresor y $61,5 \%$ en tabaquistas. Luego de un mes del inicio de los síntomas, la disnea fue relativamente frecuente: $30,7 \%$ en pacientes con antecedentes de diabetes mellitus, $38 \%$ en pacientes con HTA, $60 \%$ en EPOC, $31 \%$ en individuos con sobrepeso y $30,7 \%$ en tabaquistas. El dolor torácico y la disgeusia fueron infrecuentes como síntomas persistentes en todos estos grupos.

\section{Persistencia de los síntomas a los tres meses}

Ciento cincuenta $(48,70 \%)$ pacientes reportaron síntomas luego de tres meses del diagnóstico, siendo los más frecuentes fatiga $(22,8 \%)$, anosmia $(10,34 \%)$, mialgias $(9,9 \%)$ y cefalea $(9,48 \%)$. Ochenta y dos personas $(35,34 \%)$ no presentaron síntomas a los tres meses (Tabla 2). Al momento de llenar el formulario, 76 encuestados informaron que no habían pasado aún tres meses desde el diagnóstico.

Ninguna de las personas con tratamiento inmunosupresor reportó síntomas persistentes a los tres meses del inicio de la enfermedad. Sin embargo, en el resto de grupos de comorbilidades, la ausencia de síntomas persistentes fue reportada en desde el 7,7\% (en pacientes con tabaquismo), hasta un $23,1 \%$ (en pacientes con DBT).

\begin{tabular}{|c|c|c|c|}
\hline \multicolumn{4}{|c|}{ Tabla 2. Síntomas durante la etapa aguda de la enfermedad, al mes y a los 3 meses de la misma } \\
\hline SÍNTOMAS & $\begin{array}{c}\text { DURANTE LA } \\
\text { ENFERMEDAD (n=308) }\end{array}$ & $\begin{array}{c}\text { AL MES DEL EPISODI0 } \\
\text { DE COVID-19* (n=285) }\end{array}$ & $\begin{array}{c}\text { A LOS 3 MESES } \\
\text { DEL EPISODIO DE } \\
\text { COVID-19+ (n=232) }\end{array}$ \\
\hline DOLOR MUSCULAR & $172(55,84 \%)$ & $69(24,21 \%)$ & $23(9,9 \%)$ \\
\hline FIEBRE & $158(51,29 \%)$ & $11(3,57 \%)$ & $2(0,86 \%)$ \\
\hline CEFALEA & $154(50 \%)$ & $46(16,14 \%$ & $22(9,48 \%)$ \\
\hline TOS & $126(41 \%)$ & $55(19,29 \%)$ & $13(5,6 \%)$ \\
\hline FATIGA & $118(38,20 \%)$ & $151(52,95 \%)$ & $53(22,8 \%)$ \\
\hline ANOSMIA & $112(36,20 \%)$ & $60(21,05 \%)$ & $24(10,34 \%)$ \\
\hline ODINOFAGIA & $105(34,09 \%)$ & $26(9,12 \%)$ & $11(4,74 \%)$ \\
\hline DISGEUSIA & $78(25,32 \%)$ & $37(12,98 \%)$ & $10(4,31 \%)$ \\
\hline DIARREA & $52(16,88 \%)$ & 0 & 0 \\
\hline DISNEA & $37(12,01 \%)$ & $65(22,80 \%)$ & $14(6,03 \%)$ \\
\hline DOLOR TORÁCICO & $27(8,76 \%)$ & $24(8,42 \%)$ & $10(4,31 \%)$ \\
\hline PALPITACIONES & 0 & $38(13,33 \%)$ & $17(7,33 \%)$ \\
\hline DIFICULTAD PARA CONCENTRARSE & 0 & $39(13,68 \%)$ & $21(9,05 \%)$ \\
\hline DISFONÍA & 0 & $35(12,28 \%)$ & $13(5,60 \%)$ \\
\hline SIN SÍNTOMAS & $7(2,27 \%)$ & $11(3,85 \%)$ & $82(35,34 \%)$ \\
\hline
\end{tabular}

* El porcentaje se calculó con base en un total de 285 debido a que 23 personas manifestaron no haber cumplido aún un mes desde su episodio de COVD-19 en el momento de llenar el formulario.

† El porcentaje se calculó basado en un total de 232 personas debido a que 76 personas manifestaron no haber cumplido aún tres meses desde su episodio de COVD-19 en el momento de llenar el formulario. 


\section{Reinserción laboral y/o académica}

En lo que respecta a la reinserción laboral y/o académica, 137 personas $(45,7 \%)$ la lograron a los 10 días del diagnóstico, 94 (31,3\%) dentro del mes del alta epidemiológica, 46 (15,3\%) lo consiguieron luego del mes del alta y $23(7,7 \%)$ no habían podido reincorporarse al momento de llenar el formulario, aun cuando había pasado más de un mes del diagnóstico. De los 75 encuestados en los cuales no habían transcurrido aún tres meses del diagnóstico, 70 (93\%) ya se habían reincorporado laboralmente/académicamente. Dentro del grupo de pacientes que logró retomar sus actividades cotidianas a los 10 días de la enfermedad, la gran mayoría reportaba edad de 35 años o menor. Si bien la población mayor de 50 años de este estudio constituyó solo el 16,6\% del total, parecería haber una tendencia a retrasar el reinicio de la actividad laboral/académica con el aumento de la edad.

De las 137 personas que pudieron volver a realizar sus actividades académicas o laborales habituales a partir de los 10 días del diagnóstico, el 55,4\% (76) tenían de 18 a 35 años, el $34 \%$ (47) entre $35-50$ años, el 7,2\% (10) entre 51-65 años y el 2,9\% (4 entre 66 y 75 años. Ninguna de las personas mayores de 75 años se reincorporó a sus actividades habituales en este lapso de tiempo.

\begin{tabular}{|c|c|c|c|c|c|c|c|c|c|c|c|c|c|c|}
\hline & \multicolumn{2}{|c|}{$\begin{array}{r}\text { Asma } \\
(n=17)\end{array}$} & \multicolumn{2}{|c|}{$\begin{array}{c}\text { Diabetes } \\
(n=13)\end{array}$} & \multicolumn{2}{|c|}{$\begin{array}{c}\text { HTA } \\
(n=29)\end{array}$} & \multicolumn{2}{|c|}{$\begin{array}{l}\text { EPOC } \\
(n=5)\end{array}$} & \multicolumn{2}{|c|}{$\begin{array}{c}\text { Sobrepeso } \\
(n=71)\end{array}$} & \multicolumn{2}{|c|}{$\begin{array}{c}\text { Tratamiento } \\
\text { inmunosupresor } \\
(n=5)\end{array}$} & \multicolumn{2}{|c|}{$\begin{array}{c}\text { Tabaquismo } \\
(n=13)\end{array}$} \\
\hline & 1 mes & 3 meses & 1 mes & 3 meses & 1 mes & 3 meses & 1 mes & 3 meses & $1 \mathrm{mes}$ & 3 meses & 1 mes & 3 meses & $1 \mathrm{mes}$ & 3 meses \\
\hline Odinofagia & $\begin{array}{c}2 \\
(11,7 \%)\end{array}$ & $\begin{array}{c}3 \\
(17,6 \%)\end{array}$ & $\begin{array}{c}2 \\
(15,4 \%)\end{array}$ & $1(7,7 \%)$ & $\begin{array}{c}3 \\
(10,3 \%)\end{array}$ & $1(3,4 \%)$ & $\begin{array}{c}2 \\
(40 \%)\end{array}$ & 0 & $5(7 \%)$ & $\begin{array}{c}2 \\
(2,8 \%)\end{array}$ & 0 & 0 & $1(7,7 \%)$ & $\begin{array}{c}1 \\
(7,7 \%)\end{array}$ \\
\hline Anosmia & $1(5,8 \%)$ & 0 & $\begin{array}{c}2 \\
(15,4 \%)\end{array}$ & $1(7,7 \%)$ & $2(6,9 \%)$ & $2(6,9 \%)$ & 0 & 0 & $\begin{array}{c}16 \\
(22,5 \%)\end{array}$ & $5(7 \%)$ & $1(20 \%)$ & 0 & $3(23 \%)$ & $\begin{array}{c}1 \\
(7,7 \%)\end{array}$ \\
\hline Fatiga & $\begin{array}{c}8 \\
(47 \%)\end{array}$ & $\begin{array}{c}4 \\
(23,5 \%)\end{array}$ & $\begin{array}{c}7 \\
(54 \%)\end{array}$ & $\begin{array}{c}3 \\
(23,1 \%)\end{array}$ & $\begin{array}{c}19 \\
(65,5 \%)\end{array}$ & $\begin{array}{c}8 \\
(27,6 \%)\end{array}$ & $\begin{array}{c}5 \\
(100 \%)\end{array}$ & $\begin{array}{c}3 \\
(60 \%)\end{array}$ & $\begin{array}{c}44 \\
(62 \%)\end{array}$ & $\begin{array}{c}16 \\
(22,5 \%)\end{array}$ & $\begin{array}{c}4 \\
(80 \%)\end{array}$ & 0 & $\begin{array}{c}8 \\
(61,5 \%)\end{array}$ & $\begin{array}{c}5 \\
(38,4 \%)\end{array}$ \\
\hline Tos & $\begin{array}{c}3 \\
(17,6 \%)\end{array}$ & $\begin{array}{c}2 \\
(11,7 \%)\end{array}$ & $\begin{array}{c}3 \\
(23 \%)\end{array}$ & $\begin{array}{c}1 \\
(7,7 \%)\end{array}$ & $\begin{array}{c}4 \\
(13,8 \%)\end{array}$ & $\begin{array}{c}1 \\
(3,4 \%)\end{array}$ & $\begin{array}{c}2 \\
(40 \%)\end{array}$ & 0 & $\begin{array}{c}15 \\
(21,2 \%)\end{array}$ & $\begin{array}{c}5 \\
(7 \%)\end{array}$ & $\begin{array}{c}1 \\
(20 \%)\end{array}$ & 0 & $\begin{array}{c}4 \\
(30,7 \%)\end{array}$ & $\begin{array}{c}2 \\
(15,4 \%)\end{array}$ \\
\hline Disnea & $\begin{array}{c}3 \\
(17,6 \%)\end{array}$ & $\begin{array}{c}3 \\
(17,6 \%)\end{array}$ & $\begin{array}{c}4 \\
(30,7 \%)\end{array}$ & $\begin{array}{c}2 \\
(15,4 \%)\end{array}$ & $\begin{array}{c}11 \\
(38 \%)\end{array}$ & $\begin{array}{c}5 \\
(17,2 \%)\end{array}$ & $\begin{array}{c}3 \\
(60 \%)\end{array}$ & $\begin{array}{c}1 \\
(20 \%)\end{array}$ & $\begin{array}{c}22 \\
(31 \%)\end{array}$ & $\begin{array}{c}5 \\
(7 \%)\end{array}$ & 0 & 0 & $\begin{array}{c}4 \\
(30,7 \%)\end{array}$ & $\begin{array}{c}1 \\
(7,7 \%)\end{array}$ \\
\hline $\begin{array}{l}\text { Dolor } \\
\text { muscular }\end{array}$ & $\begin{array}{c}6 \\
(35 \%)\end{array}$ & $\begin{array}{c}2 \\
(11,7 \%)\end{array}$ & $\begin{array}{c}3 \\
(23 \%)\end{array}$ & 0 & $\begin{array}{c}6 \\
(20,7 \%)\end{array}$ & $\begin{array}{c}4 \\
(13,8 \%)\end{array}$ & $\begin{array}{c}2 \\
(40 \%)\end{array}$ & 0 & $\begin{array}{c}16 \\
(22,5 \%)\end{array}$ & $\begin{array}{c}2 \\
(2,8 \%)\end{array}$ & $\begin{array}{c}2 \\
(20 \%)\end{array}$ & 0 & $\begin{array}{c}5 \\
(38,5 \%)\end{array}$ & $\begin{array}{c}2 \\
(15,4 \%)\end{array}$ \\
\hline Disgeusia & $\begin{array}{c}1 \\
(5,8 \%)\end{array}$ & 0 & $\begin{array}{c}2 \\
(15,4 \%)\end{array}$ & $\begin{array}{c}1 \\
(7,7 \%)\end{array}$ & 0 & 0 & 0 & 0 & $\begin{array}{c}9 \\
(12,7 \%)\end{array}$ & $\begin{array}{c}1 \\
(1,4 \%)\end{array}$ & 0 & 0 & $\begin{array}{c}3 \\
(23 \%)\end{array}$ & $\begin{array}{c}2 \\
(15,4 \%)\end{array}$ \\
\hline Fiebre & $\begin{array}{c}1 \\
(5,8 \%)\end{array}$ & 0 & $\begin{array}{c}1 \\
(7,7 \%)\end{array}$ & 0 & $\begin{array}{c}3 \\
(10,3 \%)\end{array}$ & $\begin{array}{c}1 \\
(3,4 \%)\end{array}$ & $\begin{array}{c}3 \\
(60 \%)\end{array}$ & 0 & $\begin{array}{c}2 \\
(2,8 \%)\end{array}$ & 0 & 0 & 0 & $\begin{array}{c}1 \\
(7,7 \%)\end{array}$ & 0 \\
\hline Cefalea & $\begin{array}{c}3 \\
(17,6 \%)\end{array}$ & $\begin{array}{c}1 \\
(5,8 \%)\end{array}$ & $\begin{array}{c}1 \\
(7,7 \%)\end{array}$ & 0 & $\begin{array}{c}3 \\
(10,3 \%)\end{array}$ & $\begin{array}{c}1 \\
(3,4 \%)\end{array}$ & 0 & 0 & $\begin{array}{c}8 \\
(11,3 \%)\end{array}$ & $\begin{array}{c}5 \\
(7 \%)\end{array}$ & 0 & 0 & $\begin{array}{c}2 \\
(15,3 \%)\end{array}$ & $\begin{array}{c}1 \\
(7,7 \%)\end{array}$ \\
\hline Palpitaciones & $\begin{array}{c}2 \\
(11,7 \%)\end{array}$ & $\begin{array}{c}2 \\
(11,7 \%)\end{array}$ & $\begin{array}{c}2 \\
(15,4 \%)\end{array}$ & $\begin{array}{c}1 \\
(7,7 \%)\end{array}$ & $\begin{array}{c}3 \\
(10,3 \%)\end{array}$ & $\begin{array}{c}2 \\
(6,9 \%)\end{array}$ & $\begin{array}{c}1 \\
(20 \%)\end{array}$ & $\begin{array}{c}1 \\
(20 \%)\end{array}$ & $\begin{array}{c}8 \\
(11,3 \%)\end{array}$ & $\begin{array}{c}3 \\
(4,2 \%)\end{array}$ & 0 & 0 & $\begin{array}{c}3 \\
(23 \%)\end{array}$ & $\begin{array}{c}1 \\
(7,7 \%)\end{array}$ \\
\hline Disfonía & $\begin{array}{c}6 \\
(35 \%)\end{array}$ & $\begin{array}{c}1 \\
(5,8 \%)\end{array}$ & 0 & 0 & $\begin{array}{c}5 \\
(17,2 \%)\end{array}$ & $\begin{array}{c}1 \\
(3,4 \%)\end{array}$ & 0 & 0 & $\begin{array}{c}14 \\
(19,7 \%)\end{array}$ & $\begin{array}{c}7 \\
(9,8 \%)\end{array}$ & 0 & 0 & $\begin{array}{c}1 \\
(7,7 \%)\end{array}$ & 0 \\
\hline $\begin{array}{l}\text { Falta de } \\
\text { concentración }\end{array}$ & $\begin{array}{c}1 \\
(5,8 \%)\end{array}$ & $\begin{array}{c}2 \\
(11,7 \%)\end{array}$ & $\begin{array}{c}1 \\
(7,7 \%)\end{array}$ & 0 & $\begin{array}{c}3 \\
(10,3 \%)\end{array}$ & $\begin{array}{c}3 \\
(10,3 \%)\end{array}$ & 0 & 0 & $\begin{array}{c}11 \\
(15,5 \%)\end{array}$ & $\begin{array}{c}2 \\
(2,8 \%)\end{array}$ & 0 & 0 & $\begin{array}{c}1 \\
(7,7 \%)\end{array}$ & $\begin{array}{c}2 \\
(15,4 \%)\end{array}$ \\
\hline Dolor torácico & $\begin{array}{c}3 \\
(17,6 \%)\end{array}$ & $1(5,8 \%)$ & 0 & 0 & 0 & $\begin{array}{c}1 \\
(3,4 \%)\end{array}$ & 0 & 0 & $1(1,4 \%)$ & $1(1,4 \%)$ & 0 & 0 & 0 & 0 \\
\hline $\begin{array}{l}\text { Sin síntomas } \\
\text { persistentes a } \\
1 \text { y } 3 \text { meses }\end{array}$ & $\begin{array}{c}3 \\
(17,6 \%)\end{array}$ & $\begin{array}{c}2 \\
(11,7 \%)\end{array}$ & $\begin{array}{c}3 \\
(23 \%)\end{array}$ & $\begin{array}{c}3 \\
(23,1 \%)\end{array}$ & $\begin{array}{c}7 \\
(24 \%)\end{array}$ & $\begin{array}{c}4 \\
(13,8 \%)\end{array}$ & $\begin{array}{c}1 \\
(20 \%)\end{array}$ & $\begin{array}{c}1 \\
(20 \%)\end{array}$ & $\begin{array}{c}11 \\
(15,5 \%)\end{array}$ & $\begin{array}{c}14 \\
(19,7 \%)\end{array}$ & $\begin{array}{c}1 \\
(20 \%)\end{array}$ & $\begin{array}{c}3 \\
(60 \%)\end{array}$ & $\begin{array}{c}3 \\
(23 \%)\end{array}$ & $\begin{array}{c}1 \\
(7,7 \%)\end{array}$ \\
\hline $\begin{array}{l}\text { Menos de } 1 / 3 \\
\text { meses desde } \\
\text { el inicio de la } \\
\text { enfermedad }\end{array}$ & $\begin{array}{c}3 \\
(17,6 \%)\end{array}$ & $\begin{array}{c}4 \\
(23,5 \%)\end{array}$ & $\begin{array}{c}1 \\
(7,7 \%)\end{array}$ & $\begin{array}{c}3 \\
(23,1 \%)\end{array}$ & $\begin{array}{c}2 \\
(6,9 \%)\end{array}$ & $\begin{array}{c}11 \\
(38 \%)\end{array}$ & $\begin{array}{c}1 \\
(20 \%)\end{array}$ & $\begin{array}{c}2 \\
(40 \%)\end{array}$ & $\begin{array}{c}5 \\
(7 \%)\end{array}$ & $\begin{array}{c}22 \\
(31 \%)\end{array}$ & 0 & $\begin{array}{c}1 \\
(20 \%)\end{array}$ & $\begin{array}{c}1 \\
(7,7 \%)\end{array}$ & $\begin{array}{c}5 \\
(38,4 \%)\end{array}$ \\
\hline
\end{tabular}


De las 94 personas que reiniciaron sus actividades habituales dentro del mes del alta, el 31,9\% (30) tenían entre 18 y 35 años, el 43,6\% (41) entre 36 y 50 años, y el $24,4 \%$ (23) entre 51 y 65 años. Ninguna persona mayor a 75 años se reincorporó a sus actividades dentro del mes del alta.

Luego de un mes del alta, 46 personas pudieron volver a sus actividades cotidianas. El $41,3 \%$ (19) tenían entre 18 y 35 años, el $45,6 \%$ (21) entre 36 y 50 años, el $2,1 \%$ (1) entre 66 y 75 años y 2 personas (4,3\%) más de 75 años. Veintitrés personas no se habían reincorporado a trabajar en el momento de completar el formulario. De ellas, 10 tenían entre 18 y 35 años, 10 entre 36 y 50 años y una más de 75 años.

De los 122 hombres que contestaron sobre el tiempo a la reincorporación a actividades habituales, 53 de ellos $(43,44 \%)$ lo hicieron al alta, $40(32,7 \%)$ dentro del mes del alta, $19(15,5 \%)$ luego del mes del alta y $10(8,1 \%)$ no se había reincorporado aún al llenar el formulario. De las 178 mujeres que respondieron a esa misma pregunta, 84 $(47,2 \%)$ regresaron a sus actividades al alta, $54(30,3 \%)$ dentro del mes del alta, 27 (15\%) luego del mes del alta y $13(7,3 \%)$ no habían retomado actividades hasta el momento de llenar el formulario (ver Tabla 1).

\section{Reinicio de actividad física}

De los 299 sujetos que respondieron a la pregunta sobre el momento de reinicio de actividad física, 40 (17\%) lo hicieron inmediatamente al alta epidemiológica, 52 $(22,3 \%)$ dentro del mes del alta, 68 (29\%) luego del mes del alta, 73 (31\%) no habían podido iniciar aún actividad física aunque ya había pasado al menos un mes del diagnóstico. Sesenta y seis pacientes $(22,1 \%)$ reportaron no acostumbrar a hacer actividad física.

\section{Reinicio de actividades habituales y actividad física según comorbilidades}

En la Tabla 4 se muestra el tiempo de reincorporación a las actividades habituales según las comorbilidades existentes.

De los 31 hombres con antecedente de sobrepeso, el $42 \%$ se reincorporaron a sus actividades habituales al alta, el $45 \%$ dentro del mes del alta, el $6,4 \%$ después de un mes del alta y otro $6,4 \%$ no se habían reincorporado aún al momento de ser encuestados. Acerca de las 37 mujeres, el $24,3 \%$ se reincorporaron al alta, el $46 \%$ dentro del mes de alta, el $16,2 \%$ luego de un mes de alta, el 13,5\% no habían retomado actividades al momento de llenar el formulario. En cuanto al reinicio de actividad física, el $33,8 \%$ de las personas con sobrepeso la retomó luego de un mes de alta y el $16 \%$ no había podido reiniciar aún al momento de llenar la encuesta.

Hubo una tendencia a la reincorporación laboral más rápida en los hombres con antecedente de sobrepeso, en comparación con las mujeres con el mismo antecedente. Y el porcentaje de mujeres que aún no se habían reincorporado a su actividad habitual al momento de llenar el formulario es mayor con respecto al de hombres.

Acerca de las personas con antecedente de HTA, 11 de ellos ( 6 hombres y 5 mujeres) retomaron sus actividades habituales a los 10 días del diagnóstico, ocho $(5 \mathrm{H}$ y $3 \mathrm{M})$ lo

\begin{tabular}{|c|c|c|c|c|c|c|c|c|c|c|}
\hline $\begin{array}{l}\text { Tiempo de } \\
\text { reincorporación } \\
\text { a actividades } \\
\text { habituales }\end{array}$ & ASMA & Sobrepeso & Diabetes & $\begin{array}{l}\text { Tratamiento } \\
\text { inmuno- } \\
\text { supresor }\end{array}$ & HTA & EPOC & $\begin{array}{c}\text { Requerimiento } \\
\text { de diálisis }\end{array}$ & Cáncer & HIV & Tabaquismo \\
\hline $\begin{array}{l}\text { Al alta (a los } \\
10 \text { días del } \\
\text { diagnóstico) }\end{array}$ & $\begin{array}{c}7 \\
(41,2 \%)\end{array}$ & $\begin{array}{c}22 \\
(32 \%)\end{array}$ & $\begin{array}{c}3 \\
(30 \%)\end{array}$ & $\begin{array}{c}1 \\
(20 \%)\end{array}$ & $\begin{array}{c}11 \\
(38 \%)\end{array}$ & $\begin{array}{c}3 \\
(60 \%)\end{array}$ & 0 & $\begin{array}{c}1 \\
(50 \%)\end{array}$ & $\begin{array}{c}1 \\
(100 \%)\end{array}$ & $\begin{array}{c}7 \\
(53,8 \%)\end{array}$ \\
\hline $\begin{array}{l}\text { Dentro del mes } \\
\text { del alta }\end{array}$ & $\begin{array}{c}6 \\
(35,3 \%) \\
\end{array}$ & $\begin{array}{c}31 \\
(45,5 \%) \\
\end{array}$ & $\begin{array}{c}3 \\
(30 \%) \\
\end{array}$ & $\begin{array}{c}3 \\
(60 \%) \\
\end{array}$ & $\begin{array}{c}8 \\
(27,5 \%) \\
\end{array}$ & 0 & 0 & 0 & 0 & $\begin{array}{c}3 \\
(23 \%) \\
\end{array}$ \\
\hline $\begin{array}{l}\text { Luego de un } \\
\text { mes del alta }\end{array}$ & $\begin{array}{c}2 \\
(11,7 \%) \\
\end{array}$ & $\begin{array}{c}8 \\
(11,7 \%) \\
\end{array}$ & $\begin{array}{c}2 \\
(20 \%) \\
\end{array}$ & 0 & $\begin{array}{c}3 \\
(10,3 \%) \\
\end{array}$ & 0 & $\begin{array}{c}1 \\
(100 \%) \\
\end{array}$ & $\begin{array}{c}1 \\
(50 \%) \\
\end{array}$ & 0 & $\begin{array}{c}2 \\
(15,3 \%) \\
\end{array}$ \\
\hline $\begin{array}{l}\text { No se } \\
\text { reincorporó aun }\end{array}$ & \begin{tabular}{|c|}
2 \\
$(11,7 \%)$ \\
\end{tabular} & $\begin{array}{c}7 \\
(10,2 \%) \\
\end{array}$ & $\begin{array}{c}2 \\
(20 \%) \\
\end{array}$ & $\begin{array}{c}1 \\
(20 \%) \\
\end{array}$ & $\begin{array}{c}3 \\
(10,3 \%) \\
\end{array}$ & $\begin{array}{c}1 \\
(20 \%) \\
\end{array}$ & 0 & 0 & 0 & $\begin{array}{c}1 \\
(7,7 \%) \\
\end{array}$ \\
\hline Total general & 17 & 68 & 10 & 5 & 29 & 5 & 1 & 2 & 1 & 13 \\
\hline
\end{tabular}


hicieron dentro del mes del alta, tres $(1 \mathrm{H}$ y $2 \mathrm{M})$ luego de un mes del alta y tres $(1 \mathrm{H}$ y $2 \mathrm{M}$ ) no se habían reincorporado en el momento de llenar el formulario. Cuatro personas no contestaron esta pregunta. Cuando se preguntó acerca de la actividad física, el $14 \%$ pudo retomarla dentro del mes de alta o directamente al alta, el $24 \%$ luego de un mes del alta y otro $24 \%$ no había podido retomar actividad física después del episodio de COVID-19.

\section{Comorbilidades y requerimiento de internación}

De los 71 pacientes que se consideraban con sobrepeso, el $50,7 \%$ no requirió internación, el $46 \%$ estuvo internado en clínica médica y el $2,8 \%$ requirió internación en UTI. De los 35 individuos que requirieron internación, 15 de ellos (42,8\%) necesitaron oxígeno por cánula nasal o máscara, 22 (62,8\%) requirieron corticoides, 8 (22,8\%) recibieron transfusión de plasma de convalecientes, 9 $(25,7 \%)$ recibieron lopinavir/ritonavir y $2(6,2 \%)$ requirieron ventilación mecánica.

Con respecto a la necesidad de internación de los pacientes con HTA, el $55 \%$ no estuvieron internados, el $34,5 \%$ lo hicieron en clínica médica y el $10,3 \%$ en UTI.
De los 13 individuos que estuvieron internados, 8 fueron tratados con oxígeno por cánula nasal o por máscara, 2 requirieron ARM, 3 fueron tratados con lopinavir/ ritonavir, 9 recibieron corticoides y 4 recibieron plasma de convaleciente.

Debido a que solo 11 personas de la población encuestada requirieron internación en UTI, se consideró una muestra no significativa para relacionar gravedad con síntomas persistentes.

En la Tabla 5 se muestra la relación entre requerimiento de internación y persistencia de síntomas, reincorporación laboral/académica y reinicio de actividad física.

\section{Discusión}

Presentamos los resultados de un estudio sobre la persistencia de los síntomas luego de un episodio de COVID-19 y la reincorporación a las actividades laborales, académicas y físicas realizado a través de una encuesta electrónica. Observamos una persistencia de síntomas del $89 \%$ a los 30 días (fatiga y mialgias entre los más reportados) y del $34,85 \%$ a los 90 días (agregando a los

Tabla 5. Relación entre requerimiento de internación y persistencia de síntomas, reincorporación laboral/ académica y reinicio de actividad física

\begin{tabular}{|c|c|c|c|}
\hline & & $\begin{array}{l}\text { Con requerimiento } \\
\text { de internación } \\
n=100(\%)\end{array}$ & $\begin{array}{l}\text { Sin requerimiento de } \\
\text { internación } \\
n=208(\%)\end{array}$ \\
\hline \multirow[t]{2}{*}{ Síntomas persistentes al primer mes } & Sí & $94(94 \%)$ & $180(86,5 \%)$ \\
\hline & No & $6(6 \%)$ & $5(2,4 \%)$ \\
\hline \multirow[t]{2}{*}{ Síntomas persistentes al tercer mes } & Sí & $36(36 \%)$ & $114(55 \%)$ \\
\hline & No & $26(26 \%)$ & $56(27 \%)$ \\
\hline \multirow[t]{4}{*}{ Reincorporación a actividades habituales } & Al alta & $18(18 \%)$ & $125(60 \%)$ \\
\hline & Dentro del mes del alta & $43(43 \%)$ & $51(24,5 \%)$ \\
\hline & Después de 1 mes del alta & $26(26 \%)$ & $21(10,1 \%)$ \\
\hline & Aún no se reincorpora & $13(13 \%)$ & $11(5,3 \%)$ \\
\hline \multirow[t]{5}{*}{ Reinicio de actividad física } & Al alta & $2(2 \%)$ & $40(19,2 \%)$ \\
\hline & Dentro del mes del alta & $13(13 \%)$ & $40(19,2 \%)$ \\
\hline & Después de 1 mes del alta & $35(35 \%)$ & $33(15,8 \%)$ \\
\hline & Aún no reinicia & $28(28 \%)$ & $47(22,6 \%)$ \\
\hline & No realiza actividad física & $20(20 \%)$ & $47(22,6 \%)$ \\
\hline
\end{tabular}


previos anosmia). Solo el $45,7 \%$ de las personas logró la reinserción laboral/académica y el $17 \%$ de los individuos logró reiniciar actividad física al alta epidemiológica.

Nuestros datos son similares a los reportados en estudios previos. En un estudio de Goërtz et al., a los 60 días desde la aparición de los síntomas solo el $13 \%$ de los pacientes que habían sido hospitalizados por COVID-19 estaban libres de síntomas. La fatiga y la disnea fueron también los síntomas más prevalentes durante la infección y durante el seguimiento (1). En un estudio realizado en Estados Unidos, Reino Unido y Suecia sobre más de 4 millones de personas, utilizando una aplicación de teléfono inteligente, alrededor del $10 \%$ de los pacientes con infección por SARSCoV-2 permanece enfermo más allá de las tres semanas, y una proporción menor durante meses (2).

En nuestro estudio, el síntoma persistente más frecuente al mes fue fatiga, en más de la mitad de los encuestados. Otros síntomas persistentes fueron mialgias, disnea, anosmia, tos, cefalea, disgeusia, odinofagia, dolor torácico y fiebre. Un 12,9\% reportó presentar problemas para concentrarse en sus actividades habituales y el $11,5 \%$ presentó disfonía; ninguno de ellos es reconocido habitualmente como característicos del periodo "post COVID-19".

Jacobs et al. (3), en su estudio prospectivo de cohortes realizado con pacientes que habían requerido internación, describen resultados similares a los nuestros en lo relativo a los síntomas más frecuentes y a la habilidad para realizar tareas habituales. En un ensayo clínico realizado en México (4) se encontró que uno de los síntomas persistentes más frecuentes en los pacientes recuperados de COVID-19 fue la disnea y que, a diferencia de otros síntomas, no se reportó disnea en la población control que no padeció un episodio de COVID-19. En nuestro estudio, la disnea fue documentada en un $12,2 \%$ de personas durante la fase aguda de la infección, viéndose casi duplicada la frecuencia de este síntoma un mes después del diagnóstico de COVID-19. Es importante destacar que la disnea puede ser la manifestación de secuelas pulmonares, tal como han sido descritas en algunos trabajos como el de Ramani et al. (5).

Datos similares fueron señalados en el estudio de Nguyen et al. (6), en lo que se refiere a la disnea y la fatiga, agregando la pérdida de memoria, la capacidad de concentración y la caída del cabello. La persistencia de anosmia es también un denominador común en las investigaciones que se han desarrollado de síntomas post COVID-19, no siendo este trabajo la excepción. Además, debido a que se cree que es posible una inoculación intranasal de SARS-CoV-2 en el circuito olfatorio-neural, por lo que podría tener implicaciones neurodegenerativas a largo plazo, debe ser objeto de más estudios de investigación para establecer los mecanismos fisiopatológicos y las intervenciones para tratarla. Vandersteen C. et al. (7) señalan en su trabajo que la tasa de recuperación temprana (en dos meses) de la pérdida olfativa post COVID-19 constituye aproximadamente el $44 \%$ a $79 \%$ (de los cuales el $73 \%$ de los pacientes se recuperan en ocho días), lo cual coincide con los resultados de nuestro trabajo, con una persistencia de la anosmia del $21 \%$ a los 30 días y del $10,34 \%$ a los 90 días.

En los estudios de comorbilidades asociadas a la COVID-19 se ha encontrado que casi la mitad de los pacientes internados presentan comorbilidades, siendo la hipertensión la más frecuente, seguida de diabetes mellitus y enfermedad coronaria (8-11). Yang et al. (12) demostraron que entre los no sobrevivientes de un grupo de pacientes críticamente enfermos, la diabetes fue la comorbilidad predominante $(22 \%)$. Wu et al. (13), en la serie más grande reportada por el Chinese Center for Disease Control and Prevention, que incluyó 72.314 casos de COVID-19, también demostraron que los pacientes diabéticos tenían mayor mortalidad. Se cree que debido a que la diabetes mellitus generalmente conduce a un estado inflamatorio crónico de bajo grado, puede facilitar la tormenta de citoquinas, contribuyendo a presentaciones más severas del COVID-19, lo cual podría influir en el reinicio de actividades habituales más prolongado, en la persistencia de los síntomas $y$ en el aumento de la mortalidad en estos pacientes. La obesidad y la diabetes también debilitan el control y aclaramiento de los patógenos, por la disfunción del sistema inmune adaptativo.

La obesidad constituye un estado inflamatorio, asociado con mayor susceptibilidad a la infección por virus respiratorios, mayor replicación y persistencia viral, severidad de la enfermedad y a mayor complicación postinfección, falla de las vacunas y muerte.

El $35 \%$ de los pacientes con antecedentes de asma bronquial reportaron que al mes del alta presentaban disfonía o alteraciones en la voz. Este síntoma también fue reportado, con una menor prevalencia, en algunos 
pacientes con historia de HTA y sobrepeso, y en un paciente con antecedente de tabaquismo. Las afecciones posteriores al COVID-19 que afectan la producción vocal obedecen a cuatro causas principales: lesiones relacionadas con la intubación y la tos; parálisis o paresia post viral de las cuerdas vocales; neuropatía sensorial laríngea post viral y fatiga crónica (17).

Consideramos una fortaleza de este trabajo el número de personas encuestadas. Entre sus debilidades incluímos la de utilizar un formulario de opción múltiple, que fue completado por las personas interesadas en hacerlo, con respuestas que estaban condicionadas por el grado de comprensión de lo que se pretendía preguntar. Debido a que la encuesta fue enviada por redes sociales, no se dispone del número total de personas que lo recibieron y no lo contestaron. Asimismo, la mayoría de las personas que llenaron el formulario son menores de 50 años, quizás porque son aquellas que tienen más facilidad para manejar formularios en línea. Se necesita seguir estudiando la prevalencia de los síntomas y su persistencia en el tiempo para poder realizar un adecuado seguimiento a la población de personas convalecientes.

En conclusión, en nuestro estudio se observa que la persistencia de síntomas en los pacientes que han tenido un episodio de COVID-19 es frecuente, lo cual impacta negativamente en la calidad de vida. Sin embargo, pudimos observar que los síntomas fueron disminuyendo con el tiempo, lo cual puede ser un dato alentador para todos aquellos pacientes que consultan por continuar sintomáticos. Sería interesante continuar el seguimiento de estos pacientes a largo plazo para poder documentar la desaparición de toda sintomatología o, por el contrario, su persistencia en el tiempo. 


\section{Bibliografía}

1. Goërtz YMJ, Van Herck $M$, Delbressine JM, et al. Persistent symptoms 3 months after a SARSCoV-2 infection: the post-COVID-19 syndrome?. ERJ Open Res 2020; 6: 00542-2020. [https://doi. org/10.1183/23120541.00542-2020].

2. COVID Symptom Study. How Long Does COVID-19 Last? Available online at: https:// covid19.joinzoe.com/post/covid-longterm?fbclid=IwAR1RxIcmmdL-EFjh_al- .

3. Jacobs LG, GournaPaleoudis E, Lesky-Di Bari D, et al. Persistence of symptoms and quality of life at 35 days after hospitalization for COVID-19 infection. PLoSOne. 2020;15(12):e0243882. Published 2020 Dec 11. doi:10.1371/journal.pone.0243882

4. Galván-Tejada CE, Herrera-García CF, GodinaGonzález S, Villagrana-Bañuelos KE, Amaro JDL, Herrera-García K, Rodríguez-Quiñones C, ZanellaCalzada LA, Ramírez-Barranco J, Avila JLR, ReyesEscobedo F, Celaya-Padilla JM, Galván-Tejada Jl, Gamboa-Rosales H, Martínez-Acuña M, CervantesVillagrana A, Rivas-Santiago B, Gonzalez-Curiel IE. Persistence of COVID-19 Symptoms after Recovery in Mexican Population. Int J Environ ResPublicHealth. 2020 Dec 14;17(24):9367. doi: 10.3390/ijerph17249367. PMID: 33327641; PMCID: PMC7765113.

5. Ramani C, Davis Eric M , Kim J, Provencio J , Enfield J, Kadl A. Post-ICU COVID-19 Outcomes A Case Series. CHEST. January 2021

6. Nguyen $Y$, et al. Applicability of the CURB-65 pneumonia severity score for outpatient treatment of COVID-19. J infect 2020 :81 (3): e96-8.

7. Vandersteen C, Payne M, Dumas LE, Fernandez VM, Plonka A, ChirioD. Persistent olfactory complaintsafter COVID-19: a new interpretation of the psychophysical olfactory scores. Rhinology Online, Vol 4: 66 - 72, 2021. http://doi.org/10.4193/RHINOL/21.010

8. Wang $\mathrm{D}, \mathrm{Hu} \mathrm{B}, \mathrm{Hu} \mathrm{C}$, et al. ClinicalCharacteristics of 138 HospitalizedPatientsWith 2019 Novel Coronavirus-InfectedPneumonia in Wuhan, China. JAMA. 2020;323(11):1061-1069.

9. Chen N, Zhou M, Dong X, et al. Epidemiological and clinical characteristics of 99 cases of 2019 novel coronavirus pneumonia in Wuhan, China: a descriptive study. TheLancet. 2020;395(10223):507513.

10. Li B, Yang J, Zhao F, et al. Prevalence and impact of cardiovascular metabolic diseases on COVID-19 in China. Clinical research in cardiology : official journal of the German CardiacSociety. 2020.

11. Wang B, Li R, Lu Z, Huang Y. Does comorbidity increase the risk of patients with COVID-19: evidence from meta-analysis. Aging. 2020;12.

12. Yang $X, Y u Y, X u$ J, et al. Clinical course and outcomes of critically ill patients with SARS-CoV-2 pneumonia in Wuhan, China: a single-centered, retrospective, observational study. TheLancetRespiratory Medicine. 2020;8(5):475-481.

13. Wu Z, McGoogan JM. Characteristics of and ImportantLessonsFromthe Coronavirus Disease 2019 (COVID-19) Outbreak in China: Summary of a Report of 72314 Cases

14. Richardson S, Hirsch JS, Narasimhan M, et al. PresentingCharacteristics, Comorbidities, and OutcomesAmong 5700 PatientsHospitalizedWith COVID-19 in the New York City Area. Jama. 2020.

15. ICNARC reporton COVID-19 in criticalcare. 2020. www.icnarc.og.

16. Simonnet A, Chetboun M, Poissy J, et al. High prevalence of obesity in severe acute respiratory syndrome coronavirus-2 (SARS-CoV-2) requiring invasive mechanical ventilation. Obesity. $n / a(n / a)$.

17. Helding, L., Carroll, T. L., Nix, J., Johns, M. M., LeBorgne, W. D., \& Meyer, D. (2020). COVID-19 After Effects: Concerns for Singers. Journal of voice: official journal of the Voice Foundation, S0892-1997(20)30281-2. Advance online publication. https://doi.org/10.1016/j. jvoice.2020.07.032

18. Carfi A, Bernabei R, Landi F, fortheGemelliAgainst COVID-19 Post-AcuteCareStudyGroup. PersistentSymptoms in PatientsAfterAcute COVID-19. JAMA. 2020;324(6):603-605. doi:10.1001/ jama.2020.12603

19. Ngai JC, Ko FW, Ng SS, To KW, Tong M, Hui DS. The long-term impact of severe acute respiratory syndrome on pulmonary function, exercise capacity and health status. Respirology. 2010; 15:543-50. https://doi.org/10.1111/j.1440-1843.2010.01720.x PMID: 20337995

20. Ahmed H, Patel K, Greenwood DC. Long-term clinical outcomes in survivors of severe acute respiratory syndrome and Middle East respiratory syndrome coronavirus outbreaks after hospitalisation or ICU admission: A systematic review and meta-analysis. J RehabilMed. 2020 May 31; 52:jrm00063. https:// doi. org/10.2340/16501977-2694 PMID: 32449782

21. Zhou Y, Chi J, Lv W, Wang Y. Obesity and diabetes as 
high-risk factors for severe coronavirus disease 2019 (Covid-19).DiabetesMetabResRev.2021;37(2):e3377. doi:10.1002/dmrr.3377.

22. Huang $C$, Wang $Y$, Li X, et al. Clinical features of patients infected with 2019 novel coronavirus in Wuhan, China. TheLancet. 2020;395(10223):497-506. 
Characterization of persistent symptoms after SARSCoV-2 infection

Background: It is frequent that patients complain of persistent symptoms after an episode of COVID-19 infection, that worsened their quality of life

Aim: the aim of this study is to describe the main symptoms during a COVID-19 infection and persistent symptoms a month and three months after the episode. Our secondary objective is to describe the period between the COVID-19 episode and resuming work/ studies and exercise routine. Methods: this is a descriptive, observational study that included adults who underwent an episode of SARS-CoV-2 infection. Participants responded to an online survey. We include demographic and comorbidity data and symptoms during infection, and one and three months after infection. Results: 308 people responded to the survey, frequent symptoms during the episode included myalgia and fever. A month after the episode, $89 \%$ complained of persistent symptoms, most frequently myalgia and fatigue. Three months after the episode $38.85 \%$ complained of persistent symptoms, most frequently fatigue and anosmia. Only $45.7 \%$ could resume work/ studies 10 days after the diagnosis, and $17 \%$ could resume exercise routine 10 days after the diagnosis. Conclusions: there is a high percentage of people who persisted with symptoms after a COVID-19 infection. We noticed symptoms improved over time, this finding could be encouraged for patients who persist symptomatic after the episode.

Key words: post covid, SARS-CoV-2, COVID-19, persistent symptoms 